\title{
Chain flexibility and dynamics of alginate solutions in different solvents
}

\author{
Bruna Maciel $^{1} \cdot$ Claude Oelschlaeger $^{1} \cdot$ Norbert Willenbacher $^{1}$
}

Received: 23 October 2019 / Revised: 18 December 2019 / Accepted: 27 December 2019

(C) The Author(s) 2020, corrected publication 2021

\begin{abstract}
Mechanical rheometry, specifically rotational rheometry, squeeze flow, and capillary rheometry, and two microrheology methods, namely multiple-particle tracking (MPT) and diffusing wave spectroscopy (DWS) have been used to get new insight into structural and dynamical properties of alginate dissolved in solvents widely used for bioprinting, namely deionized water, phosphate-buffered saline (PBS), and Dulbecco Modified Eagle Medium (DMEM) cell media. Results demonstrate that alginate rheological properties depend on the solvent quality at concentrations higher than $1 \mathrm{wt} . \%$. In this high concentration regime, in aqueous salt-free and PBS solutions, experimental scaling exponents for the concentration dependence of the specific viscosity $\eta_{\mathrm{sp}}$ and the plateau modulus $G_{0}$ agree well with theoretical predictions for neutral polymers in good solvent whereas for the terminal relaxation time $T_{\mathrm{R}}$, the exponent is slightly higher than theoretically predicted, presumably due to the formation of aggregates. For alginate dissolved in DMEM, all exponents for $\eta_{\mathrm{sp}}, G_{0}$, and $T_{R}$ agree with predictions for polymers in theta solvents, which might be related to the formation of polyelectrolyte complex as a result of interactions between alginate and amino acids. Chain persistence length $l_{\mathrm{p}}$ values, as determined directly from high frequency rheometry for the first time, are independent of alginate concentration and temperature. Lower absolute $l_{\mathrm{p}}$ values were found for DMEM solutions compared with the other solvents. Moreover, scaling exponents for $\eta_{\mathrm{sp}}, G_{0}$, and $T_{R}$ do not change with temperature, within 20 and $60^{\circ} \mathrm{C}$. These findings suggest no change in the conformation of alginate chains with temperature.
\end{abstract}

Keywords Alginate $\cdot$ Rheology $\cdot$ Solvent quality $\cdot$ Microrheology $\cdot$ Chain flexibility

\section{Introduction}

Alginate is an anionic polysaccharide derived from marine brown algae [1]. The linear copolymer backbone is composed of consecutive blocks of $\alpha$-L-guluronic (G-blocks) and $\beta$-D-mannuronic (M-blocks) linked by a $\beta-(1-4)$ glycosidic bond. It is a biomaterial that has found numerous applications in biomedical science and engineering due to its favorable properties, including biocompatibility and ease of gelation [1]. Recently, alginate has become particularly attractive as it has been used as scaffold for cell cultivation because it can form robust cell-compatible hydrogels in physiological conditions [2]. In particular, it has been employed as bioink in a relatively novel tissue

Bruna Maciel

bruna.maciel@kit.edu

1 Institute for Mechanical Process Engineering and Mechanics, Karlsruhe Institute of Technology (KIT), 76131 Karlsruhe, Germany engineering (TE) strategy, namely the 3D bioprinting technology [3]. Bioink is one of the most important components in bioprinting and the role of the alginate is to provide a matrix scaffold to cell growth allowing reliable fabrication of $3 \mathrm{D}$ constructs after printing. Both mechanical and chemical properties of alginate in hydrogel form, obtained in presence of divalent cations, e.g., $\mathrm{Ca}^{2+}$, have been widely studied and well-established [4]. However, for better printing efficiency, properties of the hydrogel must be improved; therefore, it is very important to accurately characterize and understand properties of alginate in solution especially under physiological and cell media conditions. In the literature, however, rheological and structural properties of alginate have been mainly reported in aqueous salt-free solutions and in presence of $\mathrm{NaCl}$ [5-9]. In most of these reports, the scaling behavior of the specific viscosity $\eta_{\text {sp }}$ dependence on alginate concentration in both the dilute and semi-dilute regime has been assessed and results were compared with theoretical predictions for polyelectrolyte in salt-free solutions and in the high salt limit 
[10]. Few of them [4] also investigated the concentration dependence of the terminal relaxation time $T_{\mathrm{R}}$, which was determined to fit the shear viscosity flow curve using a generalized non-Newtonian cross model [11, 12]. To our knowledge, the concentration dependence of another key structural parameter, namely the plateau modulus $G_{0}$, has never been investigated. This quantity is directly related to the mesh size $\lambda$ of the system with $\lambda=\left(k_{\mathrm{B}} T / G_{0}\right)^{1 / 3}$ [13]. Additionally, in many studies, the persistence length $l_{\mathrm{p}}$ of alginate in dilute solutions has been determined based on intrinsic viscosity [14, 15], size-exclusion chromatography $[16,17]$ measurements, and electron microscopy images $[18,19]$. In all these studies, $l_{\mathrm{p}}$ values between 12 and 16 $\mathrm{nm}$ were found independent of the technique used and ionic strength. These above experimental data are in qualitatively good agreement with calculated values $(\sim 12 \mathrm{~nm})$ obtained from modeling simulations [20, 21]. In contrast, fluorescence correlation spectroscopy (FCS) [22] performed in the very dilute regime and small-angle X-ray scattering (SAXS) measurements [23] covering a more concentrated regime, up to $30 \mathrm{~g} / \mathrm{l}$, provide smaller $l_{p}$ values around $5 \mathrm{~nm}$ and $1-2.5 \mathrm{~nm}$, respectively. An accurate characterization of the persistence length of alginate chains is of high importance, as the chain flexibility is supposed to affect cell adhesion as suggested by Lee et al. [24].

In this study, we use classical rotational rheology, squeeze flow and capillary rheometry as well as two microrheology methods, namely multiple-particle tracking (MPT) and diffusing wave spectroscopy (DWS) to get new insight into structural and dynamical properties of bioinks composed of pure alginate dissolved in water, phosphate-buffered saline (PBS), and Dulbecco Modified Eagle Medium (DMEM) cell media. DWS allows for characterizing viscoelastic properties of alginate solutions in a very broad frequency range, from 1 to $10^{6}$ $\mathrm{rad} \mathrm{s}^{-1}$, and for the first time, we determine the persistence length $l_{\mathrm{p}}$ of latter solutions in the semi-dilute entangled regime directly from high-frequency rheological measurements. The variation of $l_{\mathrm{p}}$ with alginate concentration, type of solvent, and temperature is investigated too.

Another structural parameter investigated here is the plateau modulus $G_{0}$. This parameter is determined at intermediate frequencies by measuring it directly and we investigate its dependence on alginate concentration in a large concentration range for the three different solvents. We also determine the specific viscosity $\eta_{\mathrm{sp}}$ as well as the relaxation time $T_{\mathrm{R}}$ and establish for each parameter the scaling laws characterizing its concentration dependence. These results are compared with scaling predictions for polyelectrolytes in the salt-free and in the high salt limit [10] and for neutral polymers in good and theta solvents [25-27]. Variations of those parameters with temperature are also investigated. Table 1 below gives scaling predictions in semi-dilute unentangled and entangled solutions.

\section{Materials and methods}

\section{Sample characteristics}

Sodium alginate was purchased as alginic acid sodium salt from brown algae, BioReagent product line, from SigmaAldrich Chemie GmBH (Taufkirchen, Germany). The molar mass of each monomer, i.e., G- and M-blocks, is $198 \mathrm{~g} / \mathrm{mol}$ and the charge to charge distance in each group is $0.435 \mathrm{~nm}$ and $0.57 \mathrm{~nm}$, respectively [1]. The total molar mass determined from intrinsic viscosity measurements is in the range of $300-500 \mathrm{~kg} / \mathrm{mol}$. Phosphate buffer saline (PBS) in tablet form was purchased from Sigma-Aldrich and Dulbecco's Modified Eagle's Medium (DMEM w: $4.5 \mathrm{~g} / \mathrm{L}$ glucose, w: L-glutamine, w: sodium pyruvate, w/o: phenol red, w: $3.7 \mathrm{~g} /$ $\mathrm{L} \mathrm{NaHCO}_{3}$ ) from PAN Biotech (Aidenbach, Germany). Alginate solutions were prepared stirring the powder with three different solvents: Milli-Q water, $1 \times$ PBS $(\sim 0.15 \mathrm{M}$ salt $)$ and DMEM for approximately $1 \mathrm{~h}$ until they appeared limpid and homogeneous at concentrations that vary from 0.05 to 7 wt.\%. The $\mathrm{pH}$ value for alginate in salt-free and in PBS solutions was $7.4 \pm 0.1$, whereas alginate in DMEM solutions presented a slightly higher $\mathrm{pH}$ of $8.5 \pm 0.2$. For the sake of convenience, alginate solutions in Milli-Q water will subsequently be referred to as aqueous solutions or salt-free solutions.

\section{Rotational rheometry}

A rotational rheometer, Rheoscope I from Thermo Haake (Karlsruhe, Germany), equipped with a cone-plate measuring cell (diameter $50 \mathrm{~mm}$, cone angle $1^{\circ}$ ) was used to perform steady as well as small amplitude oscillatory shear experiments covering the frequency range from 0.1 to $100 \mathrm{rad} \mathrm{s}^{-1}$ at $20^{\circ} \mathrm{C}$ and $37^{\circ} \mathrm{C}$. Strain sweep experiments performed prior to frequency sweeps ensured that the selected strain amplitude was sufficiently small to provide a linear material response at all investigated frequencies.

\section{Oscillatory squeeze flow}

Oscillatory squeeze flow experiments were performed at 20 ${ }^{\circ} \mathrm{C}$ using a piezo-driven axial vibrator (PAV) customized at the Institute for Dynamic Material Testing (Ulm, Germany). This rheometer characterizes fluid linear viscoelastic properties in a frequency range between 0.1 and almost $10 \mathrm{kHz}$. Here, gap heights between two stainless steel plates between 18 and $43 \mu \mathrm{m}$ have been used, corresponding to very small sample volumes, typically $\sim 100 \mu \mathrm{L}$. A detailed description of the operating principles and technical details of the instrument can be found in [28]. 


\section{Capillary rheometry}

Steady shear experiments at high shear rates up to $10^{6} \mathrm{~s}^{-1}$ were performed at $20^{\circ} \mathrm{C}$ using a self-assembled capillary rheometer [29]. The samples were forced to flow through capillaries of diameter $0.5 \mathrm{~mm}$ but different lengths $(10-40 \mathrm{~mm})$ with a piston of $15 \mathrm{~mm}$ diameter at controlled volume flow rates corresponding to constant shear rate values. The resulting extrusion pressure was recorded using a pressure transducer (pressure range from 0 up to 200 bars, Industrial Sensors Incorporated, USA) and is proportional to the shear viscosity of the fluid [30].

\section{Diffusing wave spectroscopy}

Diffusing wave spectroscopy (DWS) is an extension of the traditional dynamic light scattering technique in which multiple light scattering is analyzed. This method allows for monitoring the displacement of micron-sized colloidal particles with subnanometer precision and on timescales as short as $10 \mathrm{~ns}$ so that it provides access to frequencies well above $10^{4} \mathrm{rad} \mathrm{s}^{-1}$. Due to the Brownian motion of particles, the detector records intensity fluctuations from which the intensity autocorrelation function (ICF) $\mathrm{g}_{2}(\tau)-1=\langle I(t) . I(t+\tau)\rangle_{t}$ $\langle I(t)\rangle_{t}^{2}-1$ and then the mean square displacement (MSD) $\left\langle\Delta r^{2}(t)\right\rangle$ can be calculated. Finally, both viscoelastic moduli

$G^{\prime}(\omega)$ and $G^{\prime \prime}(\omega)$ are obtained based on a generalized Stokes-Einstein equation (GSE) [31]. In our experiments, we have added polystyrene particles of diameter 300, 720, or $1400 \mathrm{~nm}$ as tracers. The choice of particle size was made to obtain the most accurate ICF in the time range investigated by our DWS setup for each alginate concentration. Additionally, prior to performing DWS measurements, we verified that no particle agglomeration occurred using light microscopy. It should also be noted that the GSE relation is valid only under the assumption that the material surrounding the sphere can be treated as an isotropic and homogeneous continuum, i.e., that the particle size is larger than the structural length scales of the probed material. For all alginate solutions investigated here, we estimated that the plateau modulus $G_{0}$ is larger than $10 \mathrm{~Pa}$. From this value, we can directly determine the mesh size $\xi$ of the network according to the classical theory of rubber elasticity assuming thermal equilibrium, namely $G_{0}=k_{\mathrm{B}} T / \xi^{3}$, where $k_{\mathrm{B}}$ is the Boltzmann constant and $T$ the temperature. We obtain a maximum mesh size of $75 \mathrm{~nm}$ and this value is much smaller than the size of the smallest tracer particles used.

A customized setup based on the DWS ResearchLab device (LS Instruments, Fribourg, Switzerland) was used. Samples were filled in standard glass cuvettes (Hellma) with a path length of $2 \mathrm{~mm}$ and a width of $5 \mathrm{~mm}$. The temperature was controlled within $\pm 0.1{ }^{\circ} \mathrm{C}$ using a temperature control chamber. The measurements were conducted at $20{ }^{\circ} \mathrm{C}$ and $37{ }^{\circ} \mathrm{C}$ and for some selected concentrations also at $60{ }^{\circ} \mathrm{C}$. A $200-\mathrm{mW}$ single-frequency laser (Torus 532, Laser Quantum) operating at a wavelength $\lambda=532 \mathrm{~nm}$ was used to illuminate the sample. We collected the transmitted light using a single-mode optical fiber and single-photon counting detector with high quantum efficiency and subsequently analyzed it employing a digital correlator. More details about the DWS device and data processing can be found in Oelschlaeger et al. [32].

From these high-frequency rheological measurements, the persistence length of viscoelastic fluids can be determined. In this regime, the stress relaxation is controlled by the internal dynamics of individual molecules and the moduli $G^{\prime}$ and $G^{\prime \prime}$ show characteristic scaling behavior:

$G^{\prime} \sim G^{\prime \prime} \sim \omega^{\alpha}$

First, the Rouse-Zimm modes dominate and $\alpha=5 / 9$. At even higher frequencies, internal bending modes of single Kuhn segments determine $G^{\prime}$ and $G^{\prime \prime}$; hence, these dynamic parameters are related to the bending modulus $\beta$ often expressed in terms of the persistence length through $\beta=$ $k_{B} T l_{p}$. In this frequency range, the scaling exponent $\alpha=3 / 4$ is found as predicted by Morse [33] and Gittes and MacKintosh [34]. The transition between these scaling regimes is marked by the inverse of the shortest Rouse relaxation time $\omega_{0}=T_{0}^{-1}$, which is directly related to the persistence length:

$\omega_{0}=\frac{k_{B} T}{8 \eta_{s} l_{p}{ }^{3}}$

where $\eta_{s}$ is the solvent viscosity.

\section{Multiple-particle tracking}

This technique allows for characterizing local mechanical and structural heterogeneities of complex materials on a micrometer length scale $[35,36]$. As both methods, DWS and bulk rheology, provide only averaged sample properties, using multiple-particle tracking (MPT), we want to verify that alginate solutions are uniform and homogeneous solutions. MPT experiments were performed using an inverted fluorescence microscope (Axio Observer D1, Zeiss), equipped with a Fluar $100 \times$, N.A. 1.3, oilimmersion lens or a C-Apochromat, $40 \times$, N.A. 1.2, water-immersion lens. In this study, we have used green fluorescent polystyrene microspheres with $0.2 \mu \mathrm{m}$ diameter (Bangs Laboratories) as tracer particles. The mixture 
Table 1 Scaling predictions for polyelectrolytes in salt-free and in the high salt limit, neutral polymers in good and theta solvents for semi-dilute unentangled and entangled solutions [10, 25-27]

\begin{tabular}{|c|c|c|c|c|c|c|}
\hline & \multicolumn{3}{|c|}{ Unentangled semi-dilute } & \multicolumn{3}{|c|}{ Entangled semi-dilute } \\
\hline & $\eta_{\mathrm{sp}}$ & $G_{0}$ & $T_{\mathrm{R}}$ & $\eta_{\mathrm{sp}}$ & $G_{0}$ & $T_{\mathrm{R}}$ \\
\hline Polyelectrolytes in salt free & $c^{0.50}$ & $c^{1}$ & $\mathrm{c}^{-0.5}$ & $\mathrm{c}^{1.5}$ & $\mathrm{c}^{1.5}$ & $c^{0}$ \\
\hline Polyelectrolytes in high salt & $\mathrm{c}^{1.25}$ & $c^{1}$ & $c^{0.25}$ & $c^{3.75}$ & $c^{2.25}$ & $c^{1.5}$ \\
\hline Neutral polymers in good solvent & $\mathrm{c}^{1.3}$ & $c^{1}$ & $c^{0.31}$ & $c^{3.75}$ & $c^{2.3}$ & $c^{1.6}$ \\
\hline Neutral polymers in theta solvent & $c^{2}$ & & $c^{1}$ & $c^{4.7}$ & $c^{2.3}$ & $c^{2.3}$ \\
\hline
\end{tabular}

containing the investigated fluid including the tracers was injected into a self-build chamber at $20^{\circ} \mathrm{C}$, consisting of a coverslip and microscope glass slide with height of $\sim 150 \mu \mathrm{m}$. Images of these fluorescent beads were recorded onto a computer via a sCMOS camera Zyla X (Andor Technology). Displacements of particle centers were monitored in a $127 \times 127 \mu \mathrm{m}$ field of view, at a rate of 50 frames/s. Movies of the fluctuating microspheres were analyzed by a custom MPT routine incorporated into the software Image Processing System (Visiometrics iPS) and a self-written Matlab program [37] based on the widely used Crocker and Grier [38] tracking algorithm. For MPT experiments, a relative experimental error of $5 \%$ was estimated.

\section{Results and discussion}

\section{Comparison of mechanical rheometry and DWS measurements}

The variation of the dynamic shear moduli $G^{\prime}$ and $G^{\prime \prime}$ as a function of frequency as determined from both mechanical and optical (DWS) rheometry are given in Fig. 1a for a $3 \mathrm{wt} . \%$ alginate aqueous solution at $20^{\circ} \mathrm{C}$. Good agreement is found between mechanical and optical methods. For both techniques, the shapes of the relaxation patterns coincide over the whole frequency range, and also the absolute values agree very well. Similar results were obtained for all systems investigated here irrespective of the type of solvent used. At low frequencies, $\omega<$ $5 \mathrm{rad} \mathrm{s}^{-1}$, the terminal flow regime with $G^{\prime} \sim \omega^{2}$ and $G^{\prime \prime} \sim \omega$ is observed and at intermediate frequencies, both moduli cross each other at $\omega=330 \mathrm{rad} \mathrm{s}^{-1}$ and a well-developed quasi-plateau in $G^{\prime}\left(G^{\prime}\right.$ increases slightly with $\left.\omega\right)$, which extends at least over one decade in frequency $\left(2 \times 10^{3}<\omega<2 \times 10^{4}\right)$ is found. At high frequencies, $\omega>5 \times 10^{4} \mathrm{rad} \mathrm{s}^{-1}$, the viscous response is first dominated by the Rouse-Zimm modes with a scaling exponent $G^{\prime \prime} \sim \omega^{5 / 9}$ and at even higher frequencies, $\omega>1.5 \times 10^{5}$ $\mathrm{rad} \mathrm{s}^{-1}$, internal bending modes of single Kuhn segments dominate with $G^{\prime \prime} \sim \omega^{3 / 4}$ as predicted theoretically. However, $G^{\prime}$ does not scale as $G^{\prime} \sim \omega^{5 / 9}$ or $G^{\prime} \sim \omega^{3 / 4}$ in the frequency range investigated here; for this parameter, the scaling occurs at higher frequencies. For the $l_{\mathrm{p}}$ calculation, the solvent contribution was subtracted from the $G^{\prime \prime}$ curve as shown in Fig. 1b. Using Eq. 2, we found $\omega_{0}=1.32 \times 10^{5} \mathrm{rad} \mathrm{s}^{-1}$ and $l_{\mathrm{p}}=15.6 \pm 3.1 \mathrm{~nm}$ for this system. The characteristic rheological parameters terminal relaxation time $T_{\mathrm{R}}$ and plateau modulus $G_{0}$ have been derived directly from the modulus curves. $T_{\mathrm{R}}$ is given by the inverse angular frequency corresponding to the first crossover of $G^{\prime}$ and $G^{\prime \prime}$. The plateau modulus $G_{0}$ is defined as the value of the modulus $G^{\prime}$ at the frequency of $\omega=10^{4} \mathrm{rad} \mathrm{s}^{-1}$ for all the investigated solutions.
Fig. 1 a Dynamic shear moduli $G^{\prime}$ and $G^{\prime \prime}$ of a $3 \mathrm{wt} . \%$ alginate aqueous solution obtained from DWS ( $G^{\prime}$ dash-dotted line, and $G^{\prime \prime}$ solid line), oscillatory squeeze flow ( $G^{\prime}$ open circles, $G^{\prime \prime}$ open squares), and rotational rheometry ( $G^{\prime}$ closed circles, G" closed squares) at $T=20{ }^{\circ} \mathrm{C}$. The modulus of water $G^{\prime \prime}=\omega \eta_{\mathrm{s}}$ is included for reference (dashed line). $\mathbf{b}$ Enlarged view of the highfrequency region. Raw DWS data (solid lines), $G^{\prime \prime}$ after subtracting water contribution (dashed line)
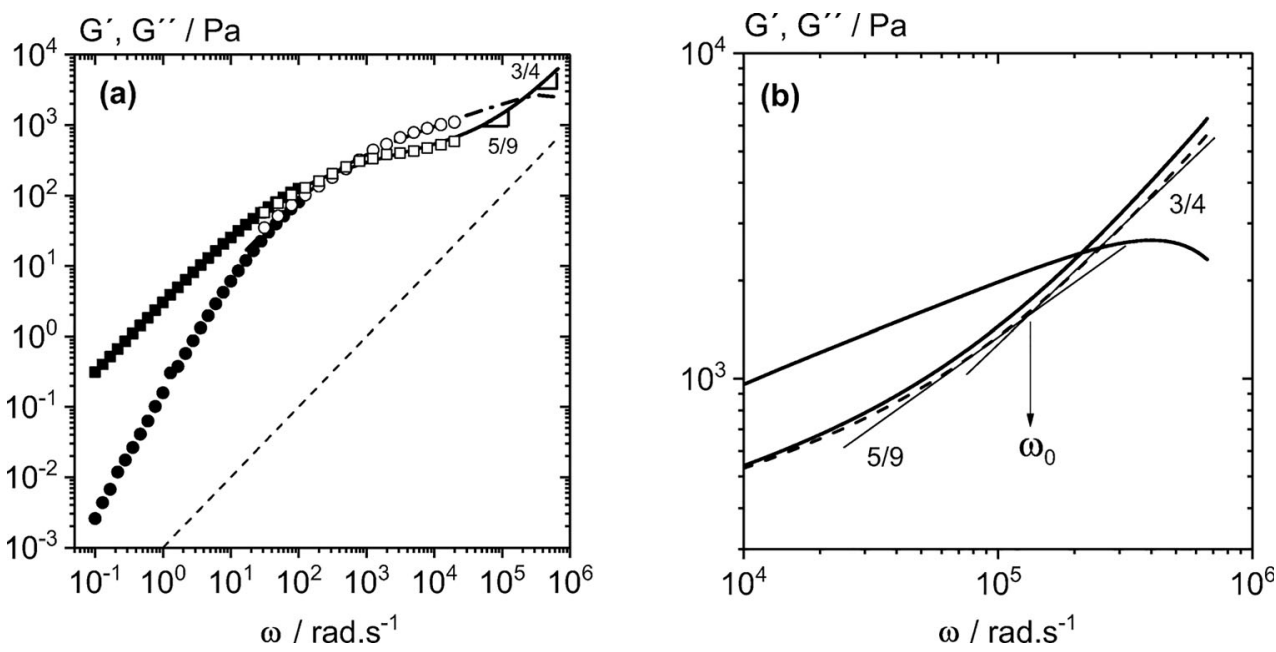


\section{Comparison of mechanical rheometry and MPT measurements}

Here, we use for the first time the multiple-particle-tracking (MPT) technique to characterize local viscoelastic properties of alginate solutions. More particularly, MPT is used to obtain information about the heterogeneity of these systems in a range of concentrations relevant for the bioprinting process, from the weakly to the highly entangled regime, i.e., from 1 to $7 \mathrm{wt} . \%$. Figure 2 shows the variation of MSDs as a function of time for PS particles of $0.2 \mu \mathrm{m}$ diameter dispersed in a slightly entangled 1 wt. $\%$ alginate aqueous solution. All MSD traces vary almost linearly with time, indicating that the motion of the tracer particles is purely diffusive and that the microenvironment surrounding the particles responds like a viscous liquid. From the averaged MSD, we determined the viscosity $\eta_{\text {MPT }}$ using the relation $\left\langle\Delta r^{2}(\tau)\right\rangle=4 D \tau$ where the StokesEinstein relation gives $D=\frac{k_{B} T}{6 \pi R \eta_{\mathrm{MPT}}}$ with $R$ being the tracer particle radius, $k_{B}$ the Boltzmann constant, and $T$ the temperature. We found $\eta_{\mathrm{MPT}}=130 \pm 40 \mathrm{mPa} . \mathrm{s}$ and within experimental error, this value is in good agreement with the viscosity value $\eta_{\text {Bulk }}=185 \pm 20 \mathrm{mPa}$.s obtained from steady shear measurements.

Additionally, the value of the non-Gaussian parameter $\alpha$, calculated for the whole ensemble of MSDs is $\alpha \approx 1$, which is a strong indicator for the homogeneity of the solution. This parameter describes the deviation of the MSD values from a Gaussian distribution expected for a homogeneous, uniform sample [39]. This quantity is zero for a Gaussian distribution, while broader distributions result in large values $\alpha \gg 0$. Similarly, low $\alpha$-values were found for all other solutions irrespective of the concentration and solvent used confirming the homogeneity of those systems. From the average MSD shown in Fig. 2, we extracted the linear viscoelastic moduli $G^{\prime}$ and $G^{\prime \prime}$ using the generalized Stokes-Einstein relation. Unphysical storage modulus values $G^{\prime}$ (not shown) are obtained due to the limitation of the Laplace transform for this weakly elastic solution and only the loss modulus $G^{\prime \prime}$ is shown. Figure 3 shows very good agreement between $G^{\prime \prime}$ data obtained from MPT and bulk rheology in the shared frequency range; as expected for a Newtonian fluid, the loss modulus increases linearly with frequency. However, for alginate solutions at concentrations $>1 \mathrm{wt} . \%$ that exhibit a significant degree of bulk elasticity $\left(G^{\prime}>100 \mathrm{~Pa}\right)$ but very short relaxation times $(<0.05 \mathrm{~s})$, MPT viscoelastic moduli cannot be determined accurately due to the limitation of the MPT technique to measure very small particle displacements. Indeed, the lower limit of accessible MSD is $\sim 10^{-4} \mu \mathrm{m}^{2}$ which corresponds to the static error of our setup [40]. Despite this limitation, we were able to determine the non-Gaussian parameter $\alpha$ for these highly concentrated solutions, but only at lag times higher than that corresponding to the static error.

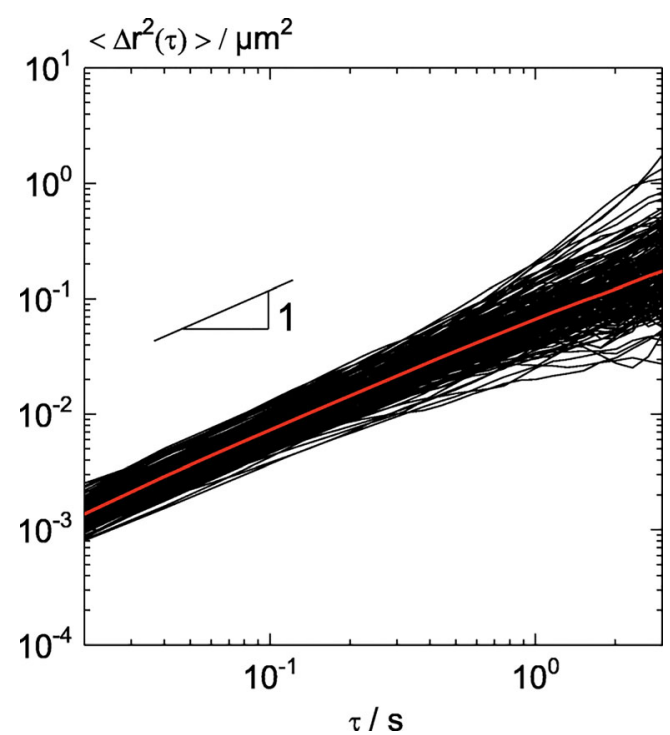

Fig. 2 MSDs of individual PS particles of diameter $0.2 \mu \mathrm{m}$ dispersed in a $1 \mathrm{wt} \%$ alginate aqueous solution. The red curve is the ensemble-average MSD

\section{Comparison of steady shear measurements, oscillatory shear, and squeeze flow rheometry as well as DWS microrheology}

In this section, we investigate the flow behavior of a $3 \mathrm{wt} . \%$ alginate aqueous solution characterized using various mechanical techniques, namely steady rotational shear and capillary rheometry, oscillatory shear and squeeze flow, as well as DWS optical microrheology. We observe very good agreement between different methods over a broad frequency/ shear rate range from 0.01 to $10^{6} \mathrm{~s}^{-1}$ as shown in Fig. 4. The complex viscosity $\left|\eta^{*}\right|$ data, obtained from oscillatory shear and squeeze flow mechanical rheometry as well as DWS

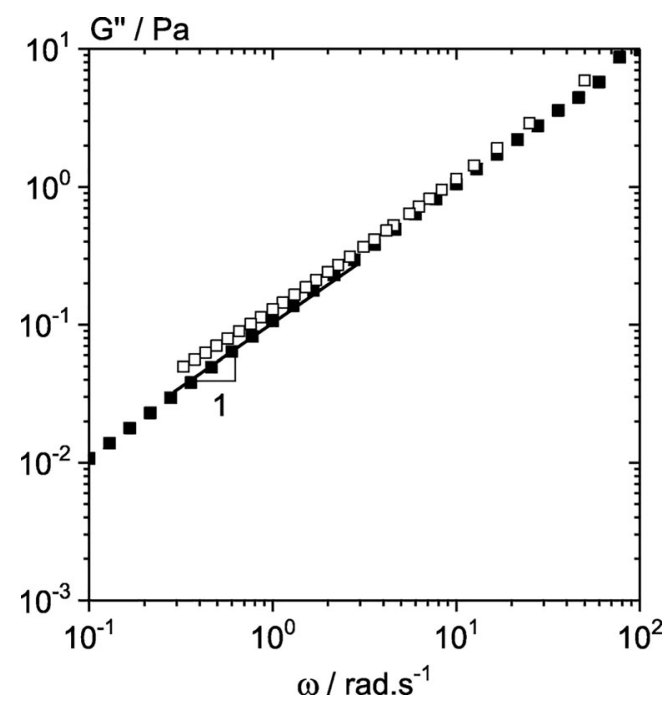

Fig. 3 Frequency-dependent viscous modulus $G^{\prime \prime}$ obtained from rotational rheometry (solid squares) and calculated from the ensembleaverage MSD (open squares) for a $1 \mathrm{wt} . \%$ alginate aqueous solution 
$\mathrm{n},\left|\mathrm{n}^{*}\right| /$ Pa.s

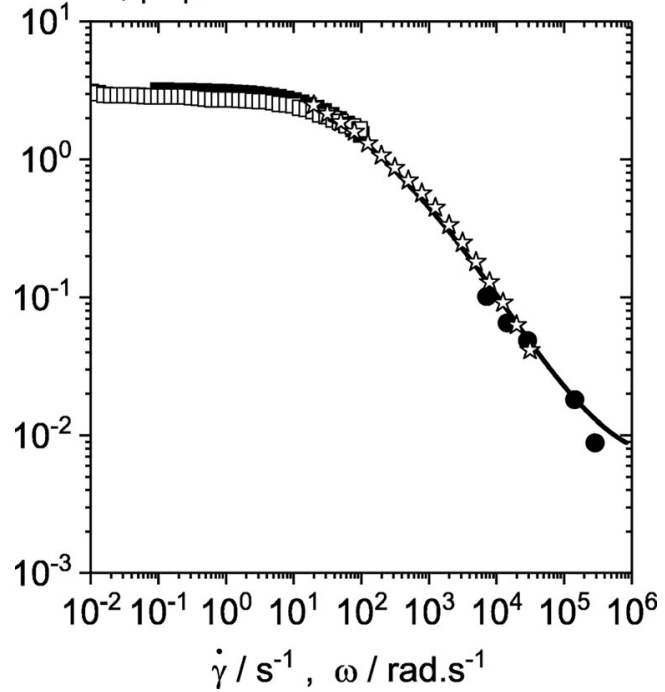

Fig. 4 Flow curve of a 3 wt.\% alginate aqueous solution at $20^{\circ} \mathrm{C}$. Steady shear viscosity from rotational (open squares) and capillary (solid circles) rheometry. Absolute value of complex viscosity from oscillatory shear (closed squares) and oscillatory squeeze (open stars) flow, as well as DWS (solid line)

microrheology, agree very well with steady shear viscosity $\eta$ data determined from rotational and capillary rheometry, demonstrating that the Cox-Merz rule is valid for the system investigated. Particularly, at high frequencies/shear rates, $\omega / \dot{\gamma}$ $>10^{3} \mathrm{~s}^{-1}$, the flow curve from DWS measurements agrees very well with the data obtained from capillary rheometry, which is the prevalent technique to measure viscosity in the high shear regime. In this case, DWS has the advantage of very low sample amount, ca. $2 \mathrm{~mL}$, whereas for capillary rheometry around $100 \mathrm{~mL}$ of material is necessary. DWS optical microrheology proves to be a suitable complementary technique to the mechanical rheometry, especially in the frequency range that rotational rheometry cannot cover. Variations of rheological parameters with alginate concentration, solvent, and temperature will be shown and discussed in the next section.

\section{Scaling behavior of the specific viscosity $\eta_{s p}$ dependence on alginate concentration}

The specific viscosity $\eta_{\mathrm{sp}}$ is defined as $\eta_{\mathrm{sp}}=\left(\eta_{0}-\eta_{\mathrm{s}}\right) \eta_{\mathrm{s}}$, where $\eta_{0}$ and $\eta_{\mathrm{s}}$ are the zero-shear viscosity and solvent viscosity, respectively, and has been determined from steady shear measurements. The concentration dependence of the specific viscosity $\eta_{\mathrm{sp}}$ for alginate in aqueous salt-free system is shown in Fig. 5. We distinguish three regimes according to the different power law dependence in concentrations $\eta_{\mathrm{sp}} \sim c^{n}$ with $n$ being the scaling exponent.

At dilute alginate concentrations ( $c<0.2 \mathrm{wt} . \%)$, we observe a power law dependence $\eta_{\mathrm{sp}} \sim c^{0.6 \pm 0.1}$ which is consistent with that expected for salt-free polyelectrolytes in the semi-dilute, unentangled regime. For $0.2 \mathrm{wt} . \%<\mathrm{c}<1 \mathrm{wt} . \%, \eta_{\mathrm{sp}} \sim c^{1.6 \pm 0.1}$ and corresponds to the behavior of salt-free polyelectrolytes in the semi-dilute, entangled regime. At higher concentrations (1 wt. $\%<\mathrm{c}<7 \mathrm{wt} . \%$ ), we found $\eta_{\mathrm{sp}} \sim c^{3.4 \pm 0.1}$; this exponent is slightly lower than the prediction for entangled solutions of neutral polymers in good solvent and polyelectrolytes in the high salt limit where $\eta_{\mathrm{sp}} \sim c^{3.75}$ [41]. Similar exponents have been obtained in all three concentration regimes in other studies on aqueous alginate solutions $[5,6]$.

Two critical concentrations are thus assigned to the two changes of slope displayed in Fig. 5, namely, $c_{e 1}$ and $c_{e 2}$. Here, $c_{e 1}$ is the concentration separating the semi-dilute unentangled and the semi-dilute entangled regime for saltfree polyelectrolytes, whereas $c_{e 2}$ marks the transition from this entangled regime for salt-free systems to the entangled regime for polyelectrolytes in the high salt limit which is similar to that of neutral polymers in good solvents. Indeed, in this highly concentrated regime, the presence of free ions in the solution screens the electrostatic interactions strongly and the behavior of the solution changes from a salt-free polyelectrolyte system to a polyelectrolyte in the high salt limit when polymer concentration is increased and their behavior then is similar to neutral polymers in good solvent. We found $c_{e 1}=$ $0.2 \mathrm{wt} . \%$, corresponding to a minimum in the reduced viscosity dependence on concentration (inset of Fig. 5) $[6,41]$ and $c_{e 2}=1 \mathrm{wt} . \%$. For the rest of the study, we will focus on the third concentration regime because the high viscosity values in this regime are required for bioprinting applications. More specifically, we will investigate the effect of alginate concentration on different rheological parameters in three different solvents: Milli-Q water, PBS, and DMEM.

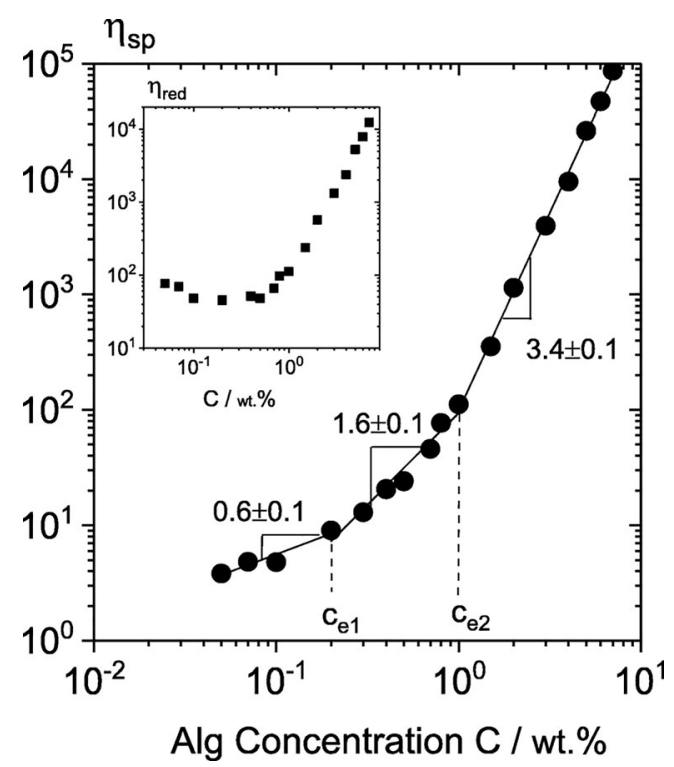

Fig. 5 Specific viscosity as a function of alginate concentration in saltfree solutions at $20^{\circ} \mathrm{C}$. Error bars are smaller than the size of the symbols. Inset shows the reduced viscosity as a function of alginate concentration 


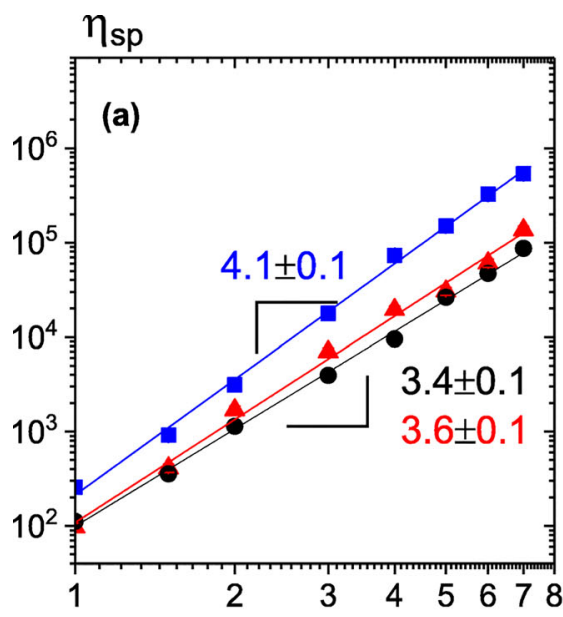

Alg Concentration / wt. \%
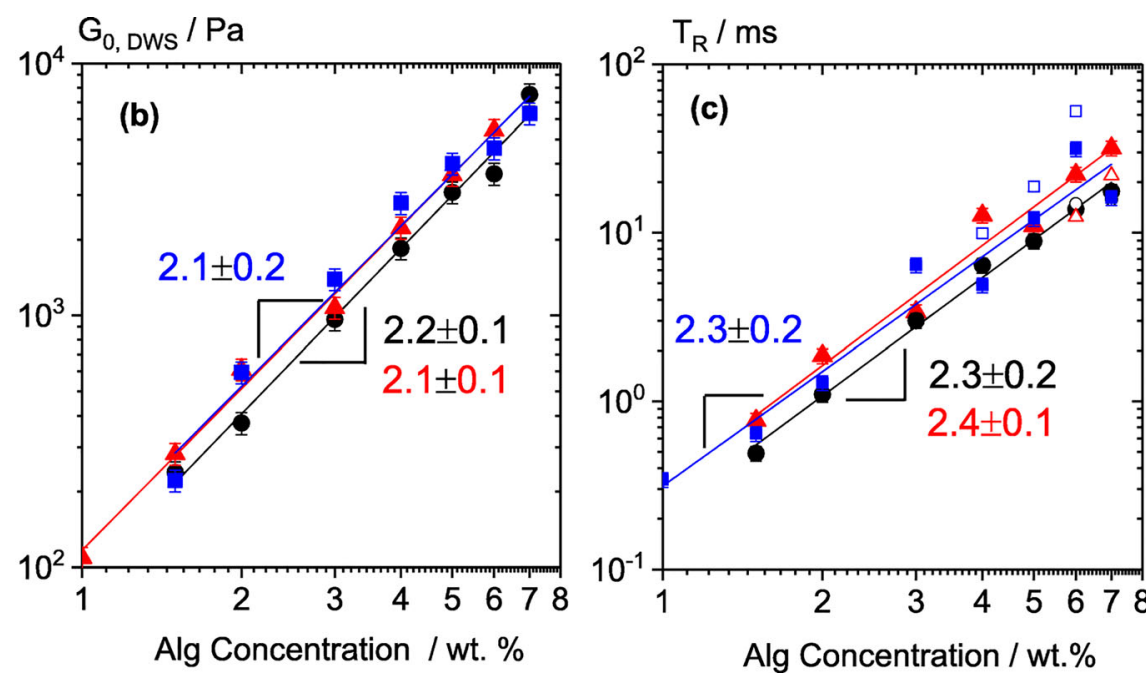

Fig. 6 Variation of the a specific viscosity, b plateau modulus, and c relaxation time as a function of alginate concentration in water (black), PBS (red), and DMEM (blue) at $20^{\circ} \mathrm{C}$. For $\mathbf{a}$ and $\mathbf{c}$, error bars are as large as the size of the symbols

\section{Effect of alginate concentration on $\eta_{s p}, G_{0}$, and $T_{R}$ in salt-free, PBS, and DMEM solutions}

Variations of $\eta_{\mathrm{sp}}, G_{0}$, and $T_{R}$ as a function of alginate concentration in the entangled regime for the three different solvents are shown in Fig. 6a, b, and c, respectively. The specific viscosity $\eta_{\text {sp }}$ has been determined from steady shear measurements, $G_{0}$ and $T_{R}$ from DWS microrheology as described above. The main advantage of the DWS technique is that it covers a very broad frequency range from low $\sim 10 \mathrm{rad} \mathrm{s}^{-1}$ to high frequency up to $10^{6} \mathrm{rad} \mathrm{s}^{-1}$ and thus allows for a determination of both parameters directly from the measured $G^{\prime}$ and $G^{\prime \prime}$ data.

As already mentioned, for alginate in aqueous salt-free solution, the specific viscosity scales as $\eta_{\mathrm{sp}} \sim c^{3.4 \pm 0.1}$ with a scaling exponent approaching the one expected for neutral polymers in good solvent. For alginate in PBS, we found a slightly higher exponent $\eta_{\mathrm{sp}} \sim c^{3.6 \pm 0.1}$, which is in very good agreement with theoretical predictions for polyelectrolytes in the high salt limit and neutral polymers in good solvent. However, for alginate in DMEM, an even stronger concentration dependence is found, $\eta_{\mathrm{sp}} \sim c^{4.1} \pm 0.1$, approaching the scaling behavior expected for neutral polymers in solvent, $\eta_{\mathrm{sp}} \sim c^{4.7}$. Other differences between salt-free, PBS and DMEM solutions reside in the absolute values of $\eta_{\mathrm{sp}}$. Compared with salt-free solutions, $\eta_{\mathrm{sp}}$ increased by a factor $\sim 2$ for solutions in PBS and by a factor of $\sim 7$ for solutions in DMEM. This difference in solvent quality might be related to the formation of polyelectrolyte complexes in DMEM solutions. DMEM contains many amino acids and these might interact with carboxylic groups from alginate to form polyelectrolyte complexes leading to stiffer gels and higher absolute values of the viscosity as compared with solutions in water or PBS. The presence of such interactions between alginate and amino acids has been proved by Di Cocco et al. [42] performing ${ }^{1} \mathrm{H}$-NMR measurements. Variation of the plateau modulus $G_{0}$ with alginate concentration in the different solvents is shown in Fig. 6b. Within experimental error, the three scaling exponent values found for this parameter agree very well and coincide with theoretical predictions for neutral polymers in good as well as in solvent $\left(G_{0} \sim c^{2.3}\right)$. For the relaxation time $T_{\mathrm{R}}$ dependence on concentration (Fig. 6c), no significant difference is observed among the solvents with $T_{\mathrm{R}} \sim c^{2.3-2.4}$. For alginate in DMEM, the scaling exponent for $T_{\mathrm{R}}$ similar as for $\eta_{\mathrm{sp}}$ and $G_{0}$ agrees well with predictions for neutral polymers in solvent, whereas for salt-free and PBS systems the exponent deviates from the prediction for neutral polymers in good solvent $\left(T_{\mathrm{R}} \sim c^{1.6}\right)$ as it has been obtained for $\eta_{\mathrm{sp}}$ and $G_{0}$. Similar unexpected $T_{\mathrm{R}}$ scaling has already been observed for hyaluronic acid

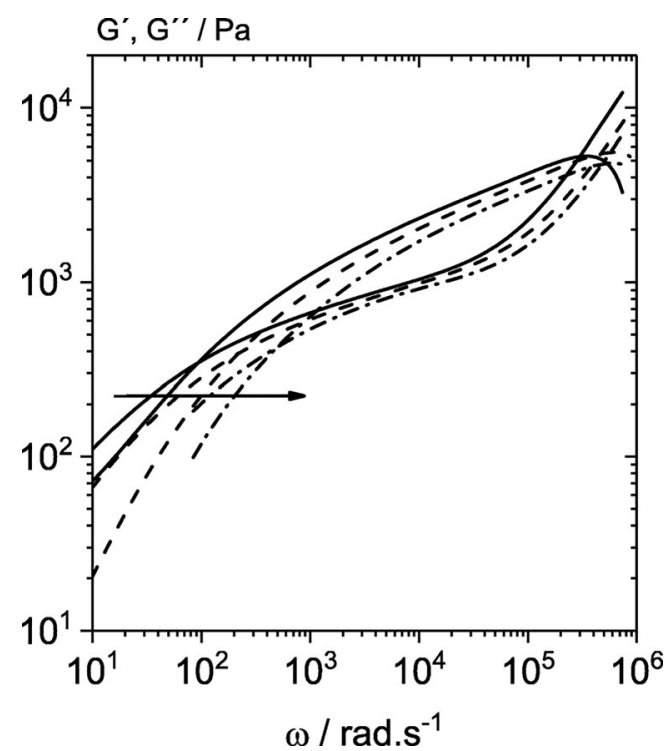

Fig. 7 Dynamic shear moduli $G^{\prime}$ and $G^{\prime \prime}$ of a 4 wt.\% alginate salt-free aqueous solution as a function of frequency obtained from DWS measurements at $20{ }^{\circ} \mathrm{C}$ (solid line), $37{ }^{\circ} \mathrm{C}$ (dashed line), and $60{ }^{\circ} \mathrm{C}$ (dash-dotted line) 
Table 2 Concentration dependence of $\eta_{\mathrm{sp}}, T_{R}$, and $G_{0}$ at $20^{\circ} \mathrm{C}$ and $37^{\circ} \mathrm{C}$ for alginate dissolved in salt-free water, PBS, and DMEM solvents

\begin{tabular}{|c|c|c|c|c|c|c|}
\hline & \multicolumn{3}{|l|}{$20^{\circ} \mathrm{C}$} & \multicolumn{3}{|l|}{$37^{\circ} \mathrm{C}$} \\
\hline & $\eta_{\mathrm{sp}}$ & $G_{0}$ & $T_{\mathrm{R}}$ & $\eta_{\mathrm{sp}}$ & $G_{0}$ & $T_{\mathrm{R}}$ \\
\hline Alginate in salt free & $c^{3.4 \pm 0.1}$ & $c^{2.2 \pm 0.1}$ & $c^{2.3 \pm 0.2}$ & $\mathrm{c}^{3.4 \pm 0.1}$ & $\mathrm{c}^{2.2 \pm 0.1}$ & $c^{2.3 \pm 0.2}$ \\
\hline Alginate in PBS & $c^{3.6 \pm 0.1}$ & $\mathrm{c}^{2.1 \pm 0.1}$ & $c^{2.4 \pm 0.1}$ & $c^{3.7 \pm 0.2}$ & $\mathrm{c}^{2.2 \pm 0.2}$ & $c^{2.1 \pm 0.1}$ \\
\hline Alginate in DMEM & $c^{4.1 \pm 0.1}$ & $\mathrm{c}^{2.1 \pm 0.2}$ & $\mathrm{c}^{2.3 \pm 0.2}$ & $c^{4.3 \pm 0.2}$ & $\mathrm{c}^{2.1 \pm 0.2}$ & $c^{2.5} \pm 0.1$ \\
\hline
\end{tabular}

[43] and alginate solutions [6]. One may attribute this deviation to the formation of aggregates or large supramolecular structures on the order of $100 \mathrm{~nm}$ at these high alginate concentrations, as it has already been observed for other polysaccharides [44]. It should be noted that relaxation time values obtained from rotational rheometry (open symbols) deviate slightly from those obtained from DWS (closed symbols) (Fig. 6c). This may be due to the inaccuracy of rotational rheology data in the frequency range $30 \mathrm{rad} \mathrm{s}^{-1}<\omega<100 \mathrm{rad} \mathrm{s}^{-1}$, which corresponds to the upper frequency limit accessible by this technique.

\section{Effect of temperature on $\eta_{s p}, G_{0}$, and $T_{R}$}

Increasing the temperature from 20 to $60^{\circ} \mathrm{C}$ decreases $\eta_{\mathrm{sp}}$ and $G_{0}$ by approximately a factor of 2 and this corresponds to a strong increase in chain mobility. Figure 7 shows modulus data for a $4 \mathrm{wt}$.\% alginate aqueous salt-free solution obtained from DWS measurements at three different temperatures 20, 37 , and $60^{\circ} \mathrm{C}$. The terminal zone and the Maxwell relaxation frequency $\omega_{\mathrm{R}}$ are strongly shifted towards higher frequencies, i.e., the relaxation time $T_{\mathrm{R}}$ decreases as expected.

The activation energy $E_{\mathrm{A}}$ required for the relaxation of the system can be calculated from the slope of the semilogarithmic plot of $T_{\mathrm{R}}$ versus of $1 / T$. We found an activation energy of $34 \mathrm{~kJ} / \mathrm{mol}$, which is in the same range as reported for other polysaccharides [43, 45].

Finally, the scaling exponents describing the concentration dependence of $\eta_{\mathrm{sp}}, T_{R}$, and $G_{0}$ are summarized in Table 2 . The scaling exponents obtained at $37^{\circ} \mathrm{C}$ are essentially the same as those obtained at $20{ }^{\circ} \mathrm{C}$ indicating no substantial change of polymer structure or conformation in this temperature range.

\section{Persistence length $I_{\mathrm{p}}$ in different solvents}

Figure 8 shows the variation of $l_{\mathrm{p}}$ as a function of alginate concentration in aqueous salt-free, PBS, and DMEM solutions as determined from $\omega_{0}$ (Eq. 2). Within experimental error, $l_{\mathrm{p}}$ is almost independent of alginate concentration for each solvent with an average value of $l_{\mathrm{p}}=15.4 \pm 0.6,15.8 \pm ; 2.5$, and 12.2 $\pm 1.5 \mathrm{~nm}$ in aqueous salt-free, PBS, and DMEM solution, respectively. The slightly lower $l_{\mathrm{p}}$ value observed in DMEM solution indicates an increase in chain flexibility which also argues for a different polymer conformation in this solvent as compared with water and PBS solutions. Similar $l_{\mathrm{p}}$ values were obtained in previous studies based on intrinsic viscosity [14] or size-exclusion chromatography [17] measurements for dilute aqueous alginate solutions in salt-free solutions and in presence of $\mathrm{NaCl}$.

The fact that the persistence length does not depend on the polysaccharide concentration in this highly concentrated regime is in good agreement with the variation predicted by the Odijk-Skolnick-Fixman (OSF) theory [46, 47]. In this theory, for polyelectrolytes, the total persistence length is defined as $l_{\mathrm{p}, T}=l_{\mathrm{p}, 0}+l_{\mathrm{p}, e}$ where $l_{\mathrm{p}, 0}$ is the intrinsic persistence length due to the rigidity of the uncharged chain and $l_{\mathrm{p}, e}$ the electrostatic persistence length contribution arising from the repulsion between adjacent ionic sites. With increasing polymer concentration and/or ionic strength, $l_{\mathrm{p}, 0}$ keeps constant whereas $l_{\mathrm{p}, e}$ decreases. The relation $l_{p, e}=1 /\left(4 \mathrm{\kappa}^{2} l_{B}\right)$ holds when the structural charge parameter $\xi>1$. This parameter is defined as the ratio of the Bjerrum length $l_{B}(0.713 \mathrm{~nm}$ in water $)$ and the distance $a$ between two adjacent ionic sites. For sodium alginate, $a=0.5 \mathrm{~nm}$ and, hence, $\xi=1.4$, which means that counterions condense. The Debye-Hückel screening length $\kappa^{-1}$ is related to the ion concentration, i.e., to the ionic force $I$ through $\mathrm{K}^{-1}=\left(4 \pi l_{B} I\right)^{-1 / 2}$. For a monovalent 1:1 electrolyte,

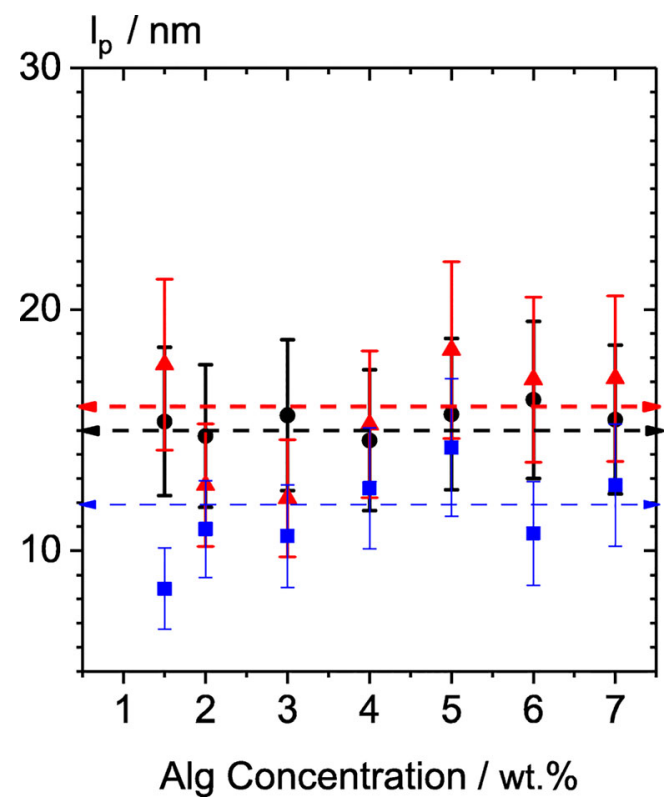

Fig. 8 Variation of the persistence length $l_{\mathrm{p}}$ as a function of alginate concentration in aqueous salt-free (black circles), PBS (red triangles), and DMEM (blue squares) solutions obtained from the crossover frequency of the $\omega^{5 / 9}$ and $\omega^{3 / 4}$ scaling regime obtained from DWS 
$I$ is identified with the free ion concentration $c_{f}=\frac{c}{\xi}+2 c_{s}$, where $c$ is the polymer concentration and $c_{s}$ is the excess salt concentration; thus, we obtain $\mathrm{K}^{2}=4 \pi l_{B} c_{f}$.

Figure 9 shows the variation of the total persistence length $l_{p, T}$ measured experimentally using DWS and the variation predicted by the OSF theory $l_{p, O S F}$ as a function of the concentration of free monovalent ions in an aqueous salt-free system. For the OSF calculation, we approximate $l_{p, T} \approx l_{p, 0}$ $=15.5 \mathrm{~nm}$ for the solution with the highest polysaccharide concentration ( $7 \mathrm{wt} . \%$ ); as for this sample, $l_{p, e}=0.16 \mathrm{~nm}$ is negligibly small.

We observe that $l_{p, T}$ is almost independent of ionic strength throughout the experimental range investigated here, consistent with the small contribution of $l_{p, e}$ decrease $(<0.75 \mathrm{~nm})$ in this concentration range as predicted by the OSF theory. The inset in Fig. 9 emphasizes this result.

Next, we have investigated the variation of $l_{\mathrm{p}}$ as a function of temperature $\left(20,37\right.$, and $\left.60{ }^{\circ} \mathrm{C}\right)$ for a $4 \mathrm{wt} . \%$ alginate sample in aqueous salt-free, PBS, and DMEM solutions (Fig. 10). In all cases, $l_{\mathrm{p}}$ is independent of temperature within experimental error, and alginate chains dissolved in DMEM are slightly more flexible compared with the solutions in PBS and deionized water at all investigated temperatures.

This indicates that the chain conformation seems not to change in this temperature range as already indicated by the temperature dependence of the $G_{0}, T_{R}$, and $\eta_{\mathrm{sp}}$ data.

\section{Conclusions}

In this work, we have used mechanical rheometry in combination with DWS and MPT optical microrheology to characterize linear viscoelastic properties of alginate in aqueous saltfree systems and for the first time also in PBS as well as in DMEM solutions in a frequency range from $10^{-1}$ to $10^{6} \mathrm{rad}$ $\mathrm{s}^{-1}$ and on a micrometer length scale. At concentrations higher than $1 \mathrm{wt} . \%$, relevant for bioprinting, alginate solutions in saltfree water and PBS exhibit scaling exponents for the concentration dependence of the specific viscosity $\eta_{\mathrm{sp}}$ and the plateau modulus $G_{0}$ that agree well with theoretical predictions for neutral polymers in good solvent. For the terminal relaxation time $T_{\mathrm{R}}$, a slightly stronger concentration dependence is observed compared with theoretical predictions, presumably due to the formation of aggregates in this high-alginate concentration regime.

For alginate dissolved in DMEM, all scaling exponents for $\eta_{\mathrm{sp}}, G_{0}$, and $T_{\mathrm{R}}$ agree with predictions for neutral polymers in theta solvents; i.e., the investigated solvents provide clearly different solvent quality. The distinctly different solvent quality of DMEM might be due to the formation of polyelectrolyte complexes in this solvent, due to interactions between alginate and amino acids present only in

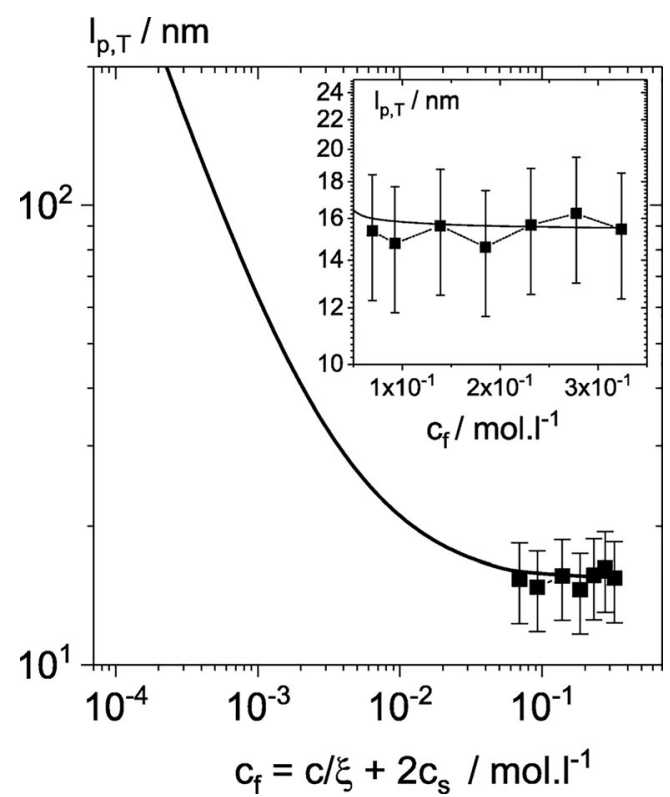

Fig. 9 Variation of the total persistence length $l_{\mathrm{p}, T}$ measured (closed squares) and calculated from OSF theory (solid line) as a function of the concentration of free monovalent ions $c_{f}$ in an aqueous alginate solution. Inset: close-up of the experimentally accessed concentration range

DMEM. This result is supported by lower persistence length values for DMEM solutions as determined directly from high-frequency rheological measurements for the first time. Whether this difference in the chain flexibility due to solvent quality affects the cell adhesion or not has to be addressed in future research. Additionally, the scaling exponents for $\eta_{\mathrm{sp}}, G_{0}$, and $T_{\mathrm{R}}$ do not change with temperature

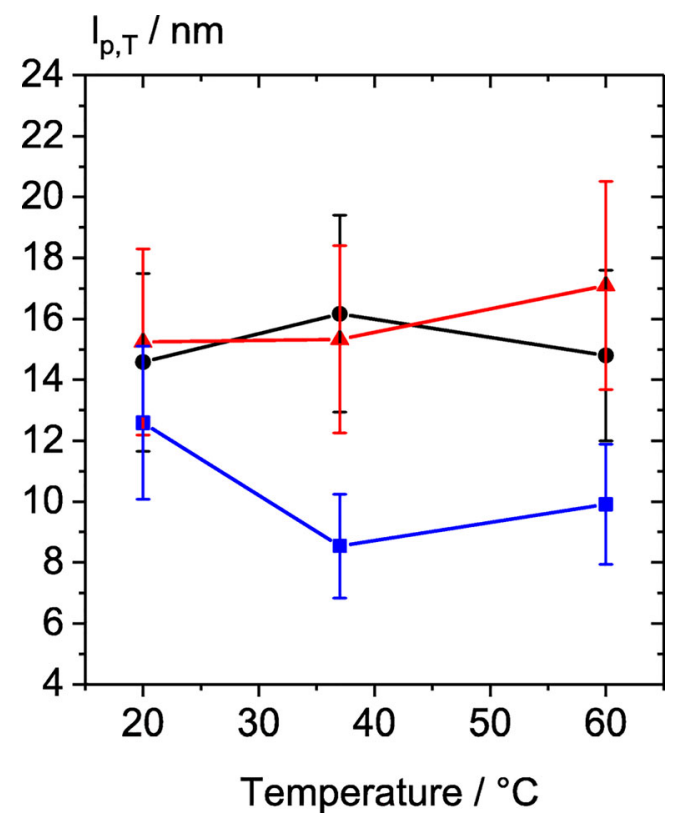

Fig. 10 Variation of the persistence length $l_{\mathrm{p}}$ as a function of temperature in aqueous salt-free (black circles), PBS (red triangles), and DMEM (blue squares) solutions 
$\left(20{ }^{\circ} \mathrm{C}<T<60{ }^{\circ} \mathrm{C}\right)$. This is also true for the observed $l_{\mathrm{p}}$ values. These findings suggest that alginate chain conformation seems not to change with temperature. This result indicates that it is possible to vary the bioink temperature during the printing process without any change of the polymer conformation. Finally, MPT results clearly reveal a homogeneous structure of alginate solutions down to the 200 -nm length scale in the concentration range investigated here.

Acknowledgments The authors thank Julian Petermann for his contribution to the DWS measurements.

Funding information Open Access funding enabled and organized by Projekt DEAL. The authors declare that the project was funded by the basic budget of our institute provided by the state ministry of science, research, and art of Baden-Württemberg.

\section{Compliance with ethical standards}

Conflict of interest The authors declare that they have no conflict of interest.

Open Access This article is licensed under a Creative Commons Attribution 4.0 International License, which permits use, sharing, adaptation, distribution and reproduction in any medium or format, as long as you give appropriate credit to the original author(s) and the source, provide a link to the Creative Commons licence, and indicate if changes were made. The images or other third party material in this article are included in the article's Creative Commons licence, unless indicated otherwise in a credit line to the material. If material is not included in the article's Creative Commons licence and your intended use is not permitted by statutory regulation or exceeds the permitted use, you will need to obtain permission directly from the copyright holder. To view a copy of this licence, visit http://creativecommons.org/licenses/by/4.0/.

\section{References}

1. Donati I, Paoletti S (2009) Material properties of alginates. In: Rehm B (ed) Alginates: biology and applications. Microbiology monographs, vol 13. Springer, Berlin

2. Yong Lee K, Mooney DJ (2012) Alginate: properties and biomedical applications. Prog Polym Sci 37:106-126

3. Selcan Gungor-Ozkerim P, Inci I, Shrike Zhang Y, Khademhosseini A, Remzi DM (2018) Bioinks for 3D bioprinting: an overview. Biomater Sci 6:915-946

4. Li L, Fang Y, Vreeker R, Appelqvist I, Mendes E (2007) Reexamining the egg-box model in calcium-alginate gels with $\mathrm{X}$ ray diffraction. Biomacromolecules 8:464-468

5. Dodero A, Vicini S, Alloisio M, Castellano M (2019) Sodium alginate solutions: correlation between rheological properties and spinnability. J Mater Sci 54:8034-8046

6. Rodríguez-Rivero C, Hilliou L, Martín del Valle EM, Galán MA (2014) Rheological characterization of commercial highly viscous alginate solutions in shear and extensional flows. Rheol Acta 53: 559-570

7. Fu S, Thacker A, Sperger DM, Boni RL, Buckner IS, Velankar S, Munson EJ, Block LH (2011) Relevance of rheological properties of sodium alginate in solution to calcium alginate gel properties. AAPS PharmSciTech 12(2):453-460

8. Bonino CA, Krebs MD, Saquing CD, Jeong SI, Shearer KL, Alsberg E, Khan SA (2011) Electrospinning alginate-based nanofibers: from blends to crosslinked low molecular weight alginateonly systems. Carbohydr Polym 85:111-119

9. Storz H, Zimmermann U, Zimmermann H, Kulicke WM (2010) Viscoelastic properties of ultra-high viscosity alginates. Rheol Acta 49:155-167

10. Dobrynin AV, Colby RH, Rubinstein M (1995) Scaling theory of polyelectrolyte solutions. Macromolecules 28:1859-1871

11. Cross MM (1965) Rheology of non-Newtonian fluids - a new flow equation for pseudoplastic systems. J Colloid Sci 20(5):417-437

12. Barnes HA (1989) Shear-thickening ("Dilatancy") in suspensions of nonaggregating solid particles dispersed in Newtonian liquids. J Rheol 33(2):329-366

13. Willenbacher N, Oelschlaeger C, Schopferer M, Fischer P, Cardinaux F, Scheffold F (2007) Broad bandwidth optical and mechanical rheometry of wormlike micelle solutions. Phys Rev Lett 99:068302

14. Zhang H, Wang H, Wang J, Guo R, Zhang Q (2001) The effect of ionic strength on the viscosity of sodium alginate solution. Polym Adv Technol 12:740-745

15. Donati I, Coslovi A, Gamini A, Skjaek-Braek G, Vetere A, Campa C, Paoletti S (2004) Galactose-substituted alginate 2: conformational aspects. Biomacromolecules 5:186-196

16. Guillermo Díaz Baños F, Díez Peña AI, Ginés Hernánez Cifre J, Carmen López Martínez M, Ortega A, de la Torre JG (2014) Influence of ionic strength on the flexibility of alginate studied by size exclusion chromatography. Carbohydr Polym 102:223-230

17. Vold IMN, Kristiansen KA, Christensen BE (2006) A study of the chain stiffness and extension of alginates, in vitro epimerized alginates, and periodate-oxidized alginates using size-exclusion chromatography combined with light scattering and viscosity detectors. Biomacromolecules 7:2136-2146

18. Stokke BT, Elgsaeter A, Skjrak-Brjek G, Smidsrød O (1987) The molecular size and shape of xanthan, xylinan, bronchial mucin, alginate, and amylose as revealed by electron microscopy. Carbohydr Res 160:13-28

19. Stokke BT, Brant DA (1990) The reliability of wormlike polysaccharide chain dimensions estimated from electron micrographs. Biopolymers 30:1161-1181

20. Braccini I, Grasso RP, Pérez S (1999) Conformational and configurational features of acidic polysaccharides and their interactions with calcium ions: a molecular modeling investigation. Carbohydr Res 317(1-4):119-130

21. Hecht H, Srebnik S (2016) Structural characterization of sodium alginate and calcium alginate. Biomacromolecules 17(6):2160 2167

22. Avaltroni F, Seijo M, Ulrich S, Stoll S, Wilkinson KJ (2007) Conformational changes and aggregation of alginic acid as determined by fluorescence correlation spectroscopy. Biomacromolecules 8:106-112

23. Josef E, Bianco-Peled H (2012) Conformation of a natural polyelectrolyte in semidilute solutions with no added salt. Soft Matter 8(35):9156-9165

24. Lee J, Song B, Subbiah R, Chung JJ, Choi UH, Park K, Kim SH, Oh SJ (2019) Effect of chain flexibility on cell adhesion: semiflexible model-based analysis of cell adhesion to hydrogels. Sci Rep 9(1):2463

25. de Gennes PG (1979) Scaling concepts in polymer physics. Cornell University Press, Ithaca

26. Colby RH, Rubinstein M, Daoud M (1994) Hydrodynamics of polymer solutions via two-parameter scaling. J Phys II 4:12991310 
27. Colby RH (2010) Structure and linear viscoelasticity of flexible polymer solutions: comparison of polyelectrolyte and neutral polymer solutions. Rheol Acta 49:425-442

28. Crassous JJ, Régisser R, Ballauff M, Willenbacher N (2005) Characterization of the viscoelastic behavior of complex fluids using the piezoelastic axial vibrator. J Rheol 49:851-863

29. Oswald W, Willenbacher N (2019) Controlling the elongational flow behavior of complex shear-thinning fluids without affecting shear viscosity. Rheol Acta. https://doi.org/10.1007/s00397-01901170-y

30. Macosko CW (1994) Rheology principles, measurements and application. Wiley-VCH, New York

31. Mason TG (2000) Estimating the viscoelastic moduli of complex fluids using the generalized Stokes-Einstein equation. Rheol Acta 39:371-378

32. Oelschlaeger C, Schopferer M, Scheffold F, Willenbacher N (2009) Linear-to-branched micelles transition: a rheometry and diffusing wave spectroscopy (DWS) study. Langmuir 25:716-723

33. Morse DC (1998) Viscoelasticity of tightly entangled solutions of semiflexible polymers. Phys Rev E 58(2):1237-1240

34. Gittes F, MacKintosh FC (1998) Dynamic shear modulus of a semiflexible polymer network. Phys Rev E 58(2):1241-1244

35. Gardel M, Valentine M, Weitz D (2005) In: Breuer KS (ed) Microscale diagnostic techniques. Springer, Heidelberg

36. Waigh TA (2005) Microrheology of complex fluids. Rep Prog Phys 68(3):685-742

37. Kowalczyk A, Oelschlaeger C, Willenbacher N (2015) Visualization of micro-scale inhomogeneities in acrylic thickener solutions: a multiple particle tracking study. Polymer 58:170-179

38. Crocker JC, Grier DG (1996) Methods of digital video microscopy for colloidal studies. J Colloid Interface Sci 179:298-310

39. Weeks ER, Crocker JC, Levitt AC, Schofield A, Weitz DA (2000) Three-dimensional direct imaging of structural relaxation near the colloidal glass transition. Science 287(5453):627-631

40. Kowalczyk A, Oelschlaeger C, Willenbacher N (2015) Tracking errors in 2D multiple particle tracking microrheology. Meas Sci Technol 26:015302

41. Rubinstein M, Colby RH, Dobrynin AV (1994) Dynamics of semidilute polyelectrolyte solutions. Phys Rev Lett 73(20):27762779

42. Di Cocco ME, Bianchetti C, Chiellini F (2003) ${ }^{1}$ HNMR studies of alginate interactions with amino acids. J Bioact Compat Polym 18: 283-296

43. Oelschlaeger C, Cota Pinto Coelho M, Willenbacher N (2013) Chain flexibility and dynamics of polysaccharide hyaluronan in entangled solutions: a high frequency rheology and diffusing wave spectroscopy study. Biomacromolecules 14(10):3689-3696

44. Esquenet C, Buhler E (2002) Aggregation behavior in semidilute rigid and semirigid polysaccharide solutions. Macromolecules 35: 3708-3716

45. Charlot A, Auzély-Velty R (2007) Novel hyaluronic acid based supramolecular assemblies stabilized by multivalent specific interactions: rheological behavior in aqueous solution. Macromolecules 40:9555-9563

46. Odijk T (1977) Polyelectrolytes near the rod limit. J Polym Sci Polym Phys Ed 15:477-483

47. Skolnick J, Fixman M (1997) Electrostatic persistence length of a wormlike polyelectrolyte. Macromolecules 10:944-948

Publisher's note Springer Nature remains neutral with regard to jurisdictional claims in published maps and institutional affiliations.

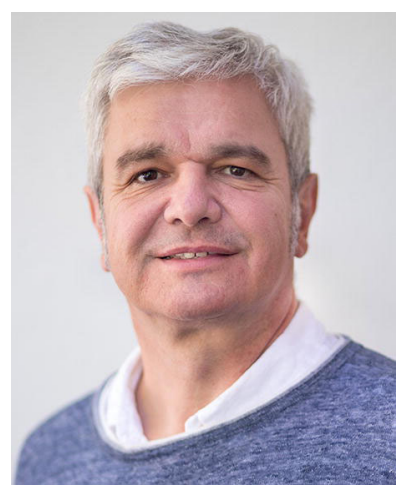

Norbert Willenbacher is head of the Institute of Mechanical Process Engineering and Mechanics at Karlsruhe Institute of Technology since 2004. He received his diploma degree in Physics and his PhD from the University of Mainz. After his dissertation at the Max Planck Institute for Polymer Research, he joined BASF SE as a research associate working on rheology of complex fluids and adhesion of soft polymers for 15 years. His research covered a broad range of complex fluids including colloidal suspensions, polymer, and surfactant solutions, as well as emulsions and foams including new measuring techniques like optical microrheology, extensional and high-frequency mechanical rheometry. Recently, he focused on rheological aspects of industrial high-speed coating processes and new biomaterials for tissue engineering and bioprinting. He pioneered the development of capillary suspensions, including the formulation of pastes for additive manufacturing of porous ceramics and highly conductive materials for soft electronics and solar cells. Prof. Willenbacher is vice-president of the German Society of Rheology. 